\title{
Low noise dual-wavelength erbium fiber laser in single-longitudinal-mode operation
}

\author{
S. Rota-Rodrigo, R.A. Perez-Herrera, M. Fernandez-Vallejo and M. López-Amo
}

Abstract-A new stable dual-wavelength fiber ring laser based on erbium doped fiber amplification is reported. The laser is based on ring resonators and employs fiber Bragg gratings to select the operation wavelengths. The topology of the laser has a significant influence in its performance: allowing an independent control of the losses and achieving a low noise configuration. As a result, it is experimentally demonstrated that both emission lines work in single-longitudinal-mode operation and the topology offers a better stability and higher optical signal-to-noise ratios than similar configurations.

Index Terms - Erbium doped fiber, fiber Bragg grating (FBG), multiwavelength lasing, single mode operation.

\section{Introduction}

Stable multiwalength single-mode erbium doped fiber ring lasers (MEDFRLs) are very attractive sources for many applications in optical fiber sensing, sensor network multiplexing schemes and instrument testing due to their advantages: simple structures, narrow linewidth, and compatibility with other optical fiber components [1,2].

To achieve multiwavelength laser operation, versatile methods based on various gain media, such as EDF amplifiers (EDFAs) [3-5], semiconductor optical amplifiers (SOAs) [6], and Raman amplifiers [7-10] have been investigated.

Unfortunately, to get stable fiber ring lasers is not a straightforward task because they are made by a rather long cavity length and consequently narrow longitudinal mode spacing. This fact contributes to generate multiple densely spaced longitudinal modes around the central lasing wavelength, thus, laser output is usually unstable because of the multimode oscillation, mode competition and mode hopping at room temperature of multiwavelength EDF lasers. This aspect can restrict their practical applications.

Montserrat Fernández-Vallejo (montserrat.fernandez@unavarra.es), Sergio Rota-Rodriguez, Rosana Perez-Herrera and M.LopezAmo

Departamento de Ingeniería Eléctrica y Electrónica

Universidad Pública de Navarra

Campus de Arrosadía

31006 Pamplona - Navarra (Spain) 
Furthermore, ring fiber lasers are also susceptible to output power instabilities. These instabilities can degrade the performance characteristics of a sensor multiplexing network based on a laser interrogation scheme. The optimization of the ring laser configuration can improve considerably the characteristics of these lasers.

To achieve single longitudinal mode (SLM) operation, several approaches have been proposed [11-14]. In [12,13] a multi-ring cavity is proposed to guarantee a SLM operation. However, it is precise to adjust properly the laser parameters. In [13] a SLM operation of a fiber ring laser is achieved with a saturable absorber. Nevertheless, the efficiency of the fiber laser is reduced with this technique. A fiber Bragg grating FabryPerot etalon can be also used to obtain SLM fiber lasers, but the spacing between the lasing wavelengths cannot be large [14].

Dual wavelength illumination is an elegant process of interrogation for such remote Fabry-Perot cavities, since it implies a less complex set up than the white light concept and offers greater flexibility of design. Therefore, a fiber Bragg grating (FBG) based dual wavelength laser can be an appealing solution for an interferometer interrogation which improves the accuracy of the measurements [15].

In this study, a stable dual-wavelength erbium doped fiber ring laser that operates in SLM condition is experimentally proposed and demonstrated. An optical signal to noise (OSNR) as high as $65 \mathrm{~dB}$ is obtained owing to the low noise configuration. Besides, the topology allows an independent control of the losses of each emission line. The time stability of the proposed fiber laser is also analyzed and discussed.

\section{Experimental setup and results}

The experimental setup of the proposed dual-wavelength erbium-doped fiber ring laser is shown in Figure 1 (a). The designed laser consists of an amplifying fiber ring cavity wherein a double FBG reflection for each wavelength is utilized. For this purpose, we included a four port circulator and a couple of FBGs and tunable FBGs for each wavelength. The FBGs were connected at port 2 of the circulator, because its bandwidth is narrower than the tunable FBGs, so most part of the Amplified Spontaneous Emission (ASE) was filtered at first reflection. The selected values of $\lambda_{1}$ and $\lambda_{2}$ were 1547 and $1550 \mathrm{~nm}$ respectively. In order to achieve an independent control of the system's equalization, the order of connection of the tunable FBGs was inverted at the port number 3 of the circulator, the $\lambda^{\prime}{ }_{1}$ wavelength was tuned at $1547 \mathrm{~nm}$ and $\lambda_{2}{ }_{2}$ to 1550 
$\mathrm{nm}$, this way the variable attenuator $1\left(\mathrm{VA}_{1}\right)$ controlled the gain at $\lambda_{1}$ and at the same mode the $\left(\mathrm{VA}_{2}\right)$ only affected to $\lambda_{2}$.

The gain of the fiber laser was provided by a 5 m-long Erbium doped fiber. A 980/1550 $\mathrm{nm}$ wavelength-division multiplexer (WDM) was used to introduce the $140 \mathrm{~mW}$ of optical pump power from a $980 \mathrm{~nm}$ laser diode at $140 \mathrm{~mW}$ of optical power. The laser output was extracted from the cavity using a 70:30 fiber coupler, with which $30 \%$ power was fed back into the ring cavity.

The circulator guided both emission lines inside the ring cavity and served also as an isolator in our lasing structure ensuring the unidirectional cavity and avoiding the undesired spatial hole-burning (SHB) effect. All the free terminations were immersed in refractive-index-matching gel to avoid undesirable reflections.

The laser output, as usual, was monitored by an optical spectrum analyzer (OSA) with highest spectral resolution of $0.01 \mathrm{~nm}$. The single-longitudinal-mode operation was verified by a heterodyne detection system, which is depicted in Figure 1 (b). Each lasing wavelength from our fiber laser was combined with the power of a commercial tunable laser source (TLS) using a $3 \mathrm{~dB}$ coupler. The TLS has a linewidth of $100 \mathrm{KHz}$ and its wavelength was placed close to the fiber laser emission wavelengths. Thus the beating signal was observed by means of an ESA, whose resolution bandwidth can be as good as $1 \mathrm{~Hz}$.

Figure 2 displays the output spectra of the proposed dual-wavelength EDF fiber ring laser scheme corresponding to the reflection bands of both FBGs when pump power is $140 \mathrm{~mW}$. The measured power of both channels was around $-10 \mathrm{dBm}$, which it is more than $65 \mathrm{~dB}$ higher than the amplified spontaneous emission (ASE) noise floor. An interesting conclusion to be drawn from figure 2 is that the proposed scheme is a very low noise topology because the ASE is filtered twice, this being a crucial advantage over some previously reported lasers in the literature [11-16].

A good stability and single-longitudinal-mode operation are requirements for EDF ring lasers for some applications. Before looking at the stability of the output power laser, let us first consider the SLM operation. 
As it was explained previously, fiber ring lasers usually support a vast number of spaced longitudinal modes. Specifically, accounting for the theory of fiber ring lasers the number of longitudinal modes within an emission line is:

Number of modes $=\frac{F W H M_{F B G}}{\Delta \lambda}$

and $\Delta \lambda$ is the mode spacing between longitudinal modes of the ring $=\frac{\lambda^{2}}{n L}$

Where: $\mathrm{n}$ is the refractive index

$\mathrm{L}$ is the ring length

$\lambda$ is the central mode wavelength

$\mathrm{FWHM}_{\mathrm{FBG}}$ is the bandwidth of the corresponding fiber Bragg grating

However, under certain conditions, the laser presents a SLM operation behavior as it is shown in Figure 3. The picture presents the results of the heterodyne detection system measured by the ESA: the beating between the tunable laser source and both emission lines when the tunable laser source was fine tuned to the first (Figure 3 (a)) and second (Figure 3 (b)) wavelength laser emission. From this, one can infer that owing to certain interaction of the seed light produced from one channel to the other and vice versa the channels multiple-longitudinal-mode oscillation is suppressed. Previous works of the authors also corroborate this fact $[11,17]$.

The output power of the proposed dual wavelength EDF ring laser was further investigated because the power of this kind of lasers usually suffers some changes with time which can restrict its applicability. Therefore, the short term stability of the proposed configuration was characterized. The instability is defined as the output power variation for a given interval of time and a specific confidence interval (CI), given as a percentage.

The laser has been tested during a period of 10 minutes at room temperature. The measured data have been stored each $15 \mathrm{~s}$ and a CL if $90 \%$ is considered. Figure 4 shows the power fluctuation for both emission lines of the fiber ring lasers. The instability was 1.1 and $1.25 \mathrm{~dB}$ for the emission line centered at $1550 \mathrm{~nm}$ and $1547 \mathrm{~nm}$ respectively. Unsurprisingly, the reduction of the number of longitudinal modes inside the cavity till the SLM operation has an outstanding positive effect in the output power variations.

\section{Conclusions}


In summary, we propose and demonstrate experimentally a new stable dual-wavelength EDF ring laser scheme The laser is based on ring resonators and employs fiber Bragg gratings to select the operation wavelengths. The topology of the laser has a significant influence in its performance: allowing an independent control of the losses and achieving a low noise configuration. As a result, it is experimentally demonstrated that both emission lines work in single-longitudinal-mode operation, showing an optical signal-to-noise ratio is as high as $65 \mathrm{~dB}$ and a power stability which varies from 1.1 to $1.25 \mathrm{~dB}$ for both emission lines.

\section{Acknowledgement}

The authors are grateful to the Spanish Government project TEC2010-20224-C02-01 and to Liekki for having supplied the erbium doped fiber used in the experiments.

\section{References}

[1] A. Bellemare, "Continuous-wave silica-based erbium-doped fibre lasers", Progress in Quantum Electronics, vol. 27, pp. 211-266, 2003.

[2] J.M. Lopez-Higuera, Hand book of optical fibre sensing technology, Wiley\&Sons, Berlin (Germany), 2002.

[3] A. Bellemare, M. Karásek, M. Rochette, S. LaRochelle and M. Têtu, "Room temperature multifrequency erbium-doped fiber lasers anchored on the ITU frequency grid", J.Lightwave Technol., vol. 18, pp. 825-831, 2000.

[4] N. Park and P.F. Wysocki, "24-line multiwavelength operation of erbium-doped fiber-ring laser", IEEE Photonics Technology Letters., vol. 8, pp. 1459-1461, 1996.

[5] L. Talaverano, S. Abad, S. Jarabo and M. López-Amo, "Multiwavelength fiber laser sources with Bragg-grating sensor multiplexing capability", J.Lightwave Technol. 19 pp. 553-558, 2001.

[6] F. Koch, P.C. Reeves-Hall, S.V. Chernikov and J.R. Taylor, "CW, multiple wavelength, room temperature, Raman fiber ring laser with external 19 channel, 10 $\mathrm{GHz}$ pulse generation in a single electro-absorption modulator", Conference on Optical Fiber Communication, Technical Digest Series., vol. 54, pp. WDD7/1-WDD7/3, Anaheim (USA), 2001.

[7] C.-. Kim, R.M. Sova, J.U. Kang and J.B. Khurgin, "Novel multi-wavelength cascaded-Raman source based on tunable fiber Sagnac loop filter," Conference on Optical Fiber Communication, Technical Digest Series., vol. 70, pp. 242-243, Anaheim (USA), (2002).

[8] Y. Han, J.H. Lee, S.H. Kim and S.B. Lee, "Tunable multi-wavelength Raman fibre laser based on fibre Bragg grating cavity with PMF Lyot-Sagnac filter", Electron.Lett. vol. 40, pp. 1475-1476, 2004.

[9] Y. Han, C. Kim, J.U. Kang, U. Paek and Y. Chung, "Multiwavelength Raman fiberring laser based on tunable cascaded long-period fiber gratings", IEEE Photonics Technology Letters., vol. 15, pp. 383-385, 2003.

[10] S. Diaz and M. Lopez-Amo, "Multiwavelength Raman fiber ring lasers with bragggrating sensor multiplexing capability", J. Op.t Commun., vol. 27, pp. 296-300, 2006.

[11] M. A. Quintela, R. A. Perez-Herrera, I. Canales, M. Fernandez-Vallejo, M. LopezAmo and J. M. Lopez-Higuera. Stabilization of dual-wavelength erbium-doped fiber 
ring lasers by single mode operation. IEEE Photonics Technology Letters, Vol. 22, No. 6, 2010.

[12] S. Pan, Z. Xiaofan and L. Caiyun, "Switchable single-longitudinal-mode dualwavelength erbium-doped fiber ring laser incorporating a semiconductor optical amplifier", Opt. Lett., vol. 33, pp. 764-766, 2008.

[13] K. Zhang and J.U. Kang, "C-band wavelength-swept single-longitudinal-mode erbium-doped fiber ring laser", Optics Express., vol. 16, pp. 14173-14179, 2008.

[14] J.L. Zhou, L. Xia, X.P. Cheng, X.P. Dong and P. Shum, "Photonic generation of tunable microwave signals by beating a dual-wavelength single longitudinal mode fiber ring laser", Appl. Phy. B., vol. 91, pp. 99-103, 2008.

[15] A.M.R. Pinto, O. Frazão, J.L. Santos, M. Lopez-Amo, J. Kobelke and K. Schuster, "Interrogation of a suspended-core Fabry-Perot temperature sensor through a dual wavelength Raman fiber laser", J. Lightwave Technol., vol. 28, pp. 3149-3155, 2010.

[16] C.H. Yeh, C.W. Chow, Y.F. Wu, Y.H. Lin, B. C. Cheng, and J. H. Chen, "Using optinal cavity loss and saturable-absorber passive filter for stable and tunable dualwavelength erbium fiber laser in single-longitudinal-mode operation", Laser Phys. Lett., $1-6,2011$.

[17] R. A. Perez-Herrera, A. Ullán, D. Leandro, M. Fernández-Vallejo, M. A. Quintela, A. Loayssa, J. M. López-Higuera and M. Lopez-Amo, "L-band multiwavelength erbium-doped fiber ring laser for sensing applications", Proceedings of SPIE - The International Society for Optical Engineering 7753, art. no. 77533C, Ottawa (Canada), 2011. 


\section{List of figure and captions:}

Figure 1. (a) Schematic diagram of the proposed dual-wavelength erbium doped fiber ring laser configuration; (b) Heterodyne detection system.

Figure 2. Output optical spectrum measured of the proposed dual-wavelength EDFRL configuration for a pump power of $140 \mathrm{~mW}$.

Figure 3. Output optical spectrum measured by the ESA for the dual-wavelength EDFRL configuration when the tunable laser was tuned to the first (a) and second (b) wavelength laser emission.

Figure 4. Output power fluctuation for both emission lines of the fiber ring lasers. 
(a)

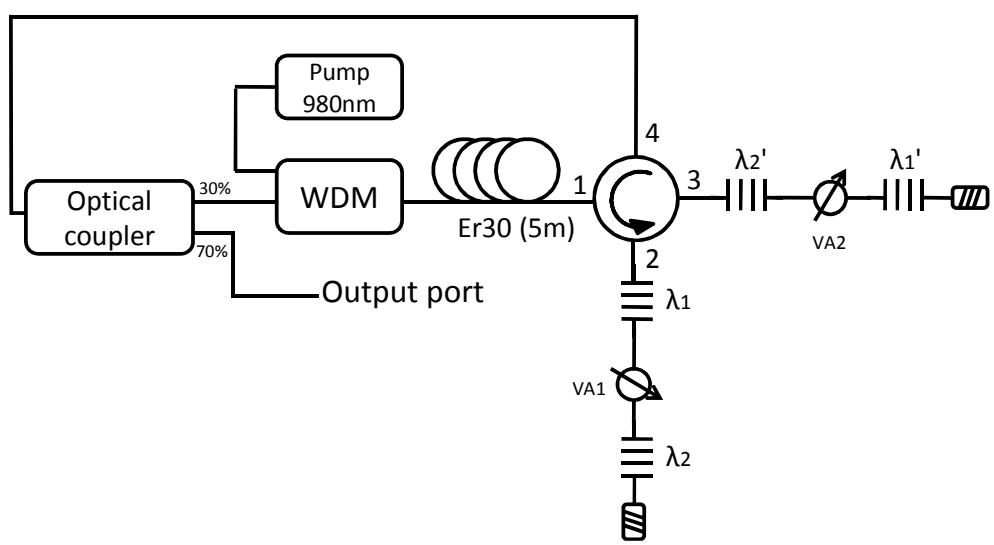

(b)

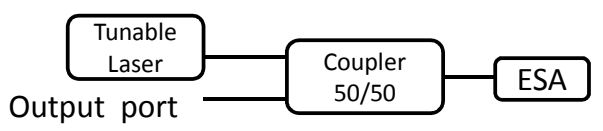

Figure 1. (a) Schematic diagram of the proposed dual-wavelength erbium doped fiber ring laser configuration; (b) Heterodyne detection system 


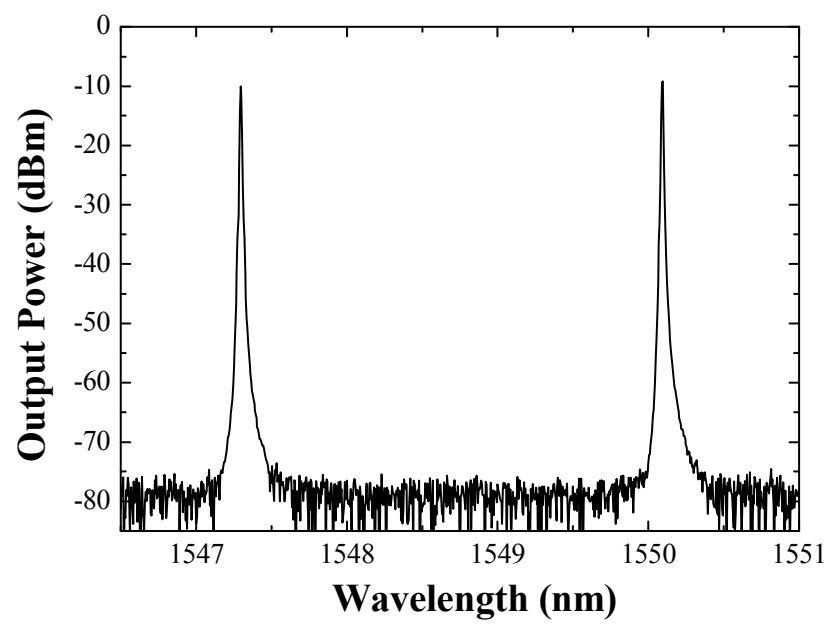

Figure 2. Output optical spectrum measured of the proposed dual-wavelength EDFRL configuration for a pump power of $140 \mathrm{~mW}$. 

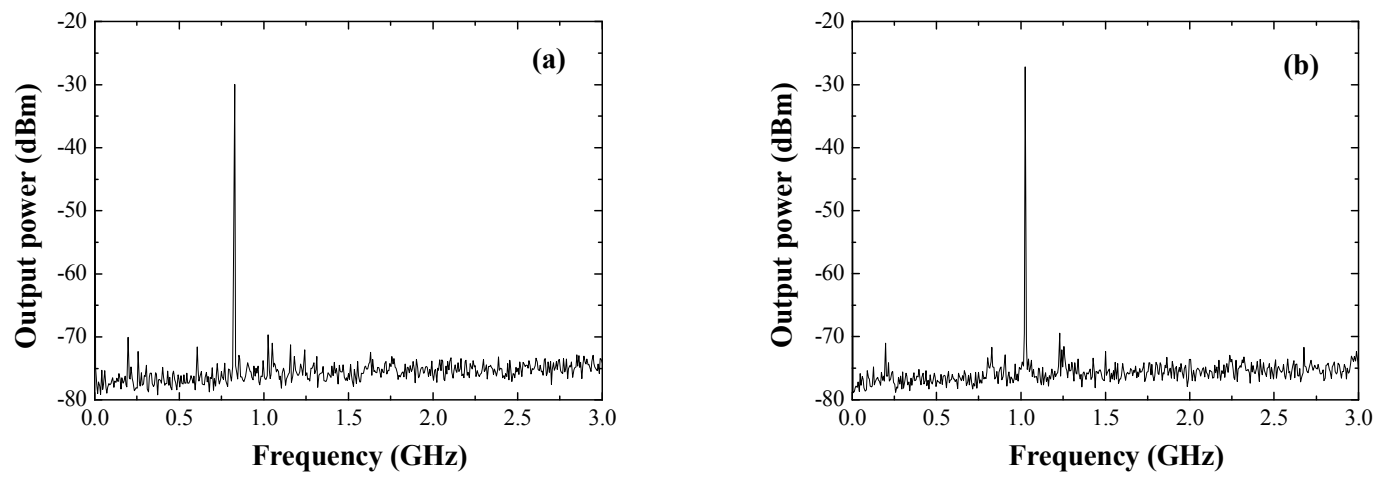

Figure 3. Output optical spectrum measured by the ESA for the dual-wavelength EDFRL configuration when the tunable laser was tuned to the first (a) and second (b) wavelength laser emission. 

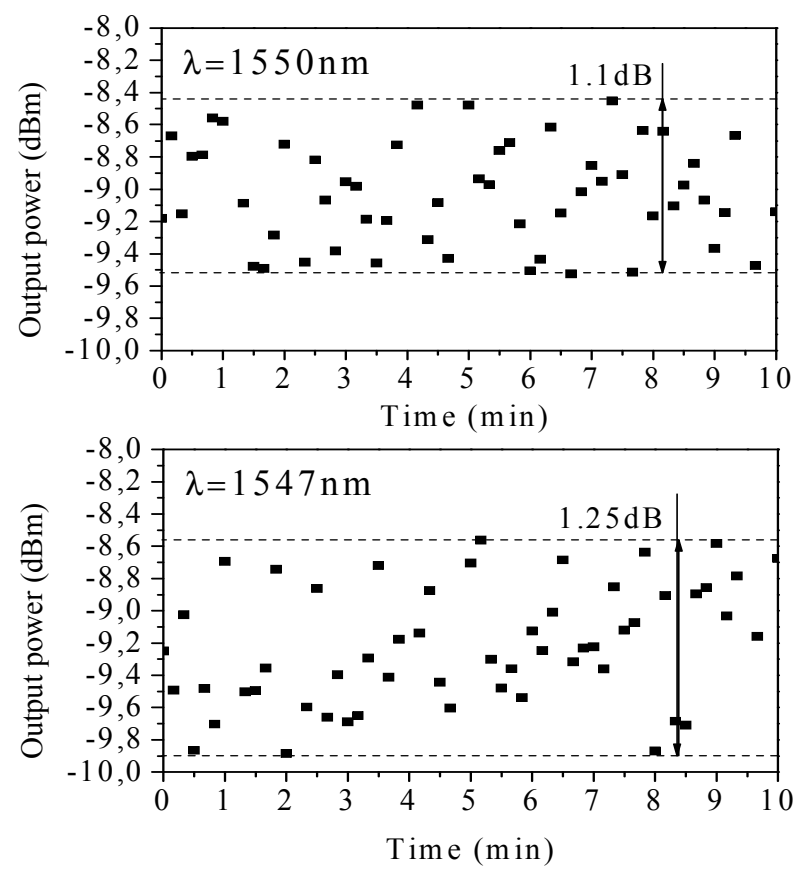

Figure 4. Output power fluctuation for both emission lines of the fiber ring lasers. 